\title{
Giulio Romani*
}

\section{A-priori bounds for quasilinear problems in critical dimension}

https://doi.org/10.1515/anona-2020-0025

Received November 1, 2018; accepted March 15, 2019.

Abstract: We establish uniform a-priori bounds for solutions of the quasilinear problems

$$
\begin{cases}-\Delta_{N} u=f(u) & \text { in } \Omega, \\ u=0 & \text { on } \partial \Omega,\end{cases}
$$

where $\Omega \subset \mathbb{R}^{N}$ is a bounded smooth convex domain and $f$ is positive, superlinear and subcritical in the sense of the Trudinger-Moser inequality. The typical growth of $f$ is thus exponential. Finally, a generalisation of the result for nonhomogeneous nonlinearities is given. Using a blow-up approach, this paper completes the results in [1, 2], extending the class of nonlinearities for which the uniform a-priori bound applies.

Keywords: Quasilinear elliptic problems; a-priori bounds; blow-up

MSC: 35J92, 35B45, 35B44

\section{Introduction and main results}

The study of a-priori bounds for solutions of elliptic boundary value problems, that is, establishing the existence of a positive constant $C$ such that $\|u\|_{L^{\infty}(\Omega)} \leq C$ for all solutions $u$, is an interesting and important issue. Indeed, on the one hand it is a key point to show existence of solutions by means of the degree theory. Moreover, a large number of different techniques have been developed to get such results for problems of the kind

$$
\begin{cases}-\Delta_{m} u=f(x, u) & \text { in } \Omega, \\ u=0 & \text { on } \partial \Omega,\end{cases}
$$

where $\Omega \subset \mathbb{R}^{N}$ is typically a smooth bounded domain, $\Delta_{m} u:=\operatorname{div}\left(|\nabla u|^{m-2} \nabla u\right)$ and $f: \Omega \times \mathbb{R} \rightarrow \mathbb{R}^{+}$has a subcritical growth in the second variable. Here subcriticality is meant in the sense of the Sobolev embeddings.

When $m=2$, namely for second-order semilinear problems, uniform a-priori bounds were firstly obtained for a (non-optimal) class of subcritical nonlinearities by Brezis and Turner in [3] by means of Hardy-Sobolev inequalities. Some years later, Gidas and Spruck in [4] and de Figueiredo, Lions and Nussbaum in [5] improved this result, applying respectively a blow-up method and a moving-planes technique together with a Pohožaev identity. The main assumption therein was that the growth at $\infty$ of $f$ is controlled by a suitable power with exponent $2^{\star}-1=\frac{N+2}{N-2}, 2^{\star}$ being the critical Sobolev exponent. We also point out the more recent work of Castro and Pardo [6] which enlarges the class of nonlinearities involved.

Regarding quasilinear equations, similar results have been achieved for $1<m<N$ by Azizieh and Clément [7], Ruiz [8], Dall'Aglio et al. [9], Lorca and Ubilla [10] and Zou [11]. In these works, the nonlinearity may depend also on $x$ and on the gradient, nonetheless its growth at infinity with respect to $u$ should be less than a subcritical power. Originally, most of these results concerned the case $1<m \leq 2$ due to some symmetry and monotonicity arguments for solutions to $m$-laplacian equations which were at that time available only

*Corresponding Author: Giulio Romani, Aix Marseille Université, CNRS, Centrale Marseille, I2M, 39 rue F. Joliot Curie, 13453 Marseille, France, E-mail: giulio.romani@univ-amu.fr 
for that range of $m$; however, those techniques have been later extended also to the case $m>2$ in the papers $[12,13]$. See also the recent work of Damascelli and Pardo [2], which further extends the class of nonlinearities for which the a-priori bound applies, in the spirit of $[5,6]$.

When, on the other hand, we consider the limiting case $m=N$, the Sobolev space $W_{0}^{1, N}(\Omega)$ compactly embeds in all Lebesgue spaces and the well-known Trudinger-Moser inequality shows that the maximal growth for a function $g$ such that $\int_{\Omega} g(u) d x<+\infty$ for any $u \in W_{0}^{1, N}(\Omega)$ is $g(t)=\exp \left\{\alpha_{N} t^{\frac{N}{N-1}}\right\}$, the constant $\alpha_{N}>0$ being explicit. Therefore, we can consider problems of kind (1) with suitable exponential nonlinearities. Nevertheless, a-priori bounds may be found only up to the threshold $t \mapsto e^{t}$, as the work [14] of Brezis and Merle shows for $m=N=2$ (see also Section 5). In addition, the authors proved local a-priori bounds when the nonlinearity is of kind $f(x, t)=V(x) e^{t}$. This, together with the boundary analysis [5], yields the desired apriori bound for convex domains. The results in [14] have been later on extended in the quasilinear setting by Aguilar Crespo and Peral Alonso in [15] and we refer also to [16] for concentration compactness issues in this direction.

The boundary value problem (1) with $m=N$ and general subcritical nonlinearities (in the sense of the Trudinger-Moser inequality) has been studied by Lorca, Ruf and Ubilla in [1], where the authors considered superlinear growths either controlled by the map $t \mapsto e^{t^{\alpha}}$ for some $\alpha \in(0,1)$, or which are comparable to $t \mapsto e^{t}$. To prove a uniform a-priori bound in the first alternative, techniques involving Orlicz spaces are applied, while for the second the authors adapt the strategy in [14].

The goal of our work is to fill the gap between these growths. Indeed, Passalacqua improved the results of [1] in his Ph.D. Thesis [17] by means of a subtle modification of the argument therein, but the gap was still not completely filled and this seems to be out of reach with those techniques. Here, instead, we apply a blow-up method inspired by [18] to deduce the desired estimates in the interior of the domain. Moreover, our results complete the analysis in [2] for the case $m=N$ and, further, to the best of our knowledge, they seem to be new even for the semilinear problem when $N=2$.

We note that a similar approach has been also applied by Mancini and the author in [19] to study higherorder problems.

Throughout all the paper, $\Omega$ is a bounded and strictly convex domain in $\mathbb{R}^{N}$ with $C^{1, \alpha}$ boundary and the nonlinearity $f$ satisfies the following conditions:

A1) $f \geq 0, f \in C^{1}(\mathbb{R})$ and $f^{\prime} \geq 0$ on $[M,+\infty)$ for some $M \geq 0$;

A2) there exists a positive constant $d$ such that $\liminf _{t \rightarrow+\infty} \frac{f(t)}{t^{N-1+d}}=+\infty$;

A3) there exists $\lim _{t \rightarrow+\infty} \frac{f^{\prime}(t)}{f(t)}:=\beta \in[0,+\infty)$.

The second assumption is standard (cf. [1, 2]) and in the linear case $N=2$ is equivalent to say that $f$ is slightly more than superlinear. Assumption (A3) is a control on the growth at $\infty$ of $f$. Indeed, Gronwall Lemma implies that for any $\varepsilon>0$ there are constants $C_{\varepsilon}, D_{\varepsilon}>0$ such that

$$
\min \left\{0, D_{\varepsilon} e^{(\beta-\varepsilon) t}-C_{\varepsilon}\right\} \leq f(t) \leq D_{\varepsilon} e^{(\beta+\varepsilon) t}+C_{\varepsilon} .
$$

We refer to [19, Lemma 1.1] for a detailed proof.

In the blow-up analysis, it will be useful to distinguish between the following cases.

Definition 1.1. Let $f$ satisfy (A1)-(A3). We say that $f$ is subcritical if

$$
\lim _{t \rightarrow+\infty} \frac{f^{\prime}(t)}{f(t)}=0
$$

On the other hand, we say that $f$ is critical if

$$
\lim _{t \rightarrow+\infty} \frac{f^{\prime}(t)}{f(t)} \in(0,+\infty)
$$


Remark 1. In view of (2), an example of a subcritical nonlinearity $f(t)=\log ^{\tau}(1+t) t^{p} e^{t^{\alpha}}$ with $\tau \geq 0, p \geq 1$ and $\alpha \in[0,1)$, and for a critical nonlinearity is $f(t)=\frac{e^{\gamma t}}{(t+1)^{q}}$ with $\gamma>0$. Notice that the second nonlinearity was excluded by the previous results $[1,17]$ e.g. when $\gamma=1, q \geq 1$.

Although this distinction will be relevant in the proof, the critical case being more delicate, our main result applies for both classes of nonlinearities.

Theorem 1.1. Let $\Omega \subset \mathbb{R}^{N}, N \geq 2$, be a bounded strictly convex domain of class $C^{1, \alpha}$ for some $\alpha \in(0,1)$ and let $f$ satisfy (A1)-(A3). Then, there exists a constant $C>0$ such that all weak solutions $u \in W_{0}^{1, N}(\Omega)$ of the problem

$$
\begin{cases}-\Delta_{N} u=f(u) & \text { in } \Omega, \\ u=0 & \text { on } \partial \Omega,\end{cases}
$$

satisfy $\|u\|_{L^{\infty}(\Omega)} \leq C$.

We recall that $u \in W_{0}^{1, N}(\Omega)$ is a weak solution of problem (3) if

$$
\int_{\Omega}|\nabla u|^{N-2} \nabla u \nabla \varphi=\int_{\Omega} f(u) \varphi, \quad \text { for all } \varphi \in C_{0}^{\infty}(\bar{\Omega}) .
$$

Notice that, unlike [1, 2], we are not prescribing that our solutions are in $L^{\infty}(\Omega)$. Indeed, by [17, Proposition 6.2.2], every $W_{0}^{1, N}(\Omega)$ weak solution of problem (3) is classical, namely it belongs to $C^{1, \gamma}(\bar{\Omega})$ for some $\gamma \in(0,1)$ (see also [9, Proposition 3.1]).

Remark 2. By the regularity theory of quasilinear problems by [20, 21] the $C^{1, \alpha}$ assumptions on $\partial \Omega$ are sufficient to guarantee that the uniform a-priori estimate in Theorem 1.1 is actually in $C^{1, \tilde{\alpha}}(\bar{\Omega})$ for some $\tilde{\alpha} \in(0,1)$.

Remark 3. We may set our problem in the framework of entropy solutions and obtain the same result. Indeed, one may prove that, under conditions (A1)-(A3), such solutions are indeed weak and in turn classical. For further details, we refer to Section 5, in particular to Remark 6.

Once the uniform a-priori bound of Theorem 1.1 is established, the existence of a weak solution for problem (3) may be proved by means of the topological degree theory, provided a control of the behaviour of $f$ in 0 is imposed.

Proposition 1.2. In addition of the assumptions of Theorem 1.1, suppose that

$$
\limsup _{t \rightarrow 0^{+}} \frac{f(t)}{t^{N-1}}<\lambda_{1},
$$

where $\lambda_{1}$ denotes the first eigenvalue of $-\Delta_{N}$. Then, problem (3) admits a positive weak solution.

We will not give the proof of Theorem 1.2, being rather standard once Theorem 1.1 is established, addressing the interested reader to [2, Theorem 1.5].

This paper is organized as follows: in Section 2 we collect some auxiliary results as well as boundary and energy estimates obtained in [1]; Section 3 contains the proof of Theorem 1.1 distinguishing between the subcritical and the critical case. In Section 4 we give a generalisation of our result for a class of nonhomogeneous problems and finally in Section 5 we provide an example of nonlinearities which, although being subcritical in the sense of Trudinger-Moser, do not satisfy assumption (A3) and for which one can find unbounded solutions.

\section{Preliminary estimates}

In this section, we state some known results which will be used in the sequel. We begin with a result by Serrin, improved by the regularity theory from [20]. Next, we state a Harnack-type inequality. 
Lemma 2.1 ([22], Theorem 2). Let $u$ be a weak solution of $-\Delta_{N} u=h$ in $B_{2 R}(0) \subset \Omega$. Then,

$$
\|u\|_{C^{1, \alpha}\left(B_{R}(0)\right)} \leq C\left(\|u\|_{L^{N}\left(B_{2 R}(0)\right)}+K R\right),
$$

for a suitable $\alpha \in(0,1)$ and positive constants $C\left(\alpha, R,\|h\|_{L^{N-\varepsilon}(\Omega)}\right)$ and $K\left(R,\|h\|_{L^{N-\varepsilon}(\Omega)}\right)$, for some $\varepsilon \in(0,1]$.

Lemma 2.2 ([22], Theorem 6). Let $u \geq 0$ be a solution of $-\Delta_{N} u=h$ in $B_{3 R}(0) \subset \Omega$. Then

$$
\max _{B_{R}(0)}(u) \leq C\left(\min _{B_{R}(0)}(u)+K\right),
$$

where the constants $C, K$ depending on $R$ and on $\|h\|_{L^{\frac{N}{N-\varepsilon}(\Omega)}}, \varepsilon \in(0,1]$.

The next result is taken from [1, Propositions 2.1 - 3.1] and concerns the behaviour near the boundary of solutions of (3) and their energy.

Proposition 2.3. There exist positive constants $r, C_{0}$ such that every solution $u \in W_{0}^{1, N}(\Omega)$ of (3) verifies

$$
u(x) \leq C_{0} \quad \text { and }|\nabla u(x)| \leq C_{0}, \quad x \in \Omega_{r},
$$

where $\Omega_{r}:=\{x \in \Omega \mid d(x, \partial \Omega) \leq r\}$, and

$$
\int_{\Omega} f(u) \leq \Lambda
$$

Roughly speaking, the proof of the first inequality in (5) is obtained similarly to the analogous estimate in the second-order case (see [5]) by means of a Picone inequality originally proved in [23] (see also [2, Theorem 2.5]). This implies the second inequality in (5) by standard regularity estimates. Finally, (6) follows from (5) testing the equation with a suitable function with vanishing gradient outside $\Omega_{r}$.

We point out that the uniform estimates (5)-(6) do not rely on assumption (A3), so they can be deduced for any superlinear growth (in the sense of assumption $(A 2)$ ).

\section{Uniform bounds inside the domain}

The argument is based on a blow-up method inspired by [18] and developed in [19]: supposing the existence of an unbounded sequence of solutions, we define a rescaled sequence which locally converges to a solution of a Liouville's equation on $\mathbb{R}^{N}$. Then, we have to distinguish two cases according to the growth of $f$. If $f$ is subcritical, the right-hand side of the limit equation is constant and this readily yields the contradiction. In the critical framework, the Liouville's equation is still nonlinear, so a more accurate analysis is needed: we will see that the energy concentrates around the blow-up points with a threshold which is too large for the energy bound of Proposition 2.3 to hold.

Let $\left(u_{k}\right)_{k}$ be a sequence of solutions of (3) and suppose there exist points $\left(x_{k}\right)_{k} \subset \Omega$ such that

$$
u_{k}\left(x_{k}\right)=\left\|u_{k}\right\|_{L^{\infty}(\Omega)}=: M_{k} \pi+\infty .
$$

Since $\Omega$ is bounded, then, up to a subsequence, $x_{k} \rightarrow x_{\infty} \in \Omega$, where $d\left(x_{\infty}, \partial \Omega\right)>0$ by Proposition 2.3. Moreover, we define $v_{k}: \Omega_{k} \rightarrow \mathbb{R}^{-}$as

$$
v_{k}(x):=u_{k}\left(x_{k}+\mu_{k} x\right)-M_{k},
$$

where $\Omega_{k}:=\frac{\Omega-x_{k}}{\mu_{k}}$ and

$$
\mu_{k}:=\frac{1}{\left(f\left(M_{k}\right)\right)^{1 / N}} .
$$


Notice that $\mu_{k} \rightarrow 0$ by (A1)-(A2) and this implies $\Omega_{k} \pi \mathbb{R}^{N}$, since $x_{\infty} \in \Omega$. Moreover, each $v_{k}$ satisfies (in a weak sense) the equation

$$
-\Delta_{N} v_{k}(x)=\frac{f\left(u_{k}\left(x_{k}+\mu_{k} x\right)\right)}{f\left(M_{k}\right)} \in[0,1], \quad x \in \Omega_{k} .
$$

Indeed, for any test function $\varphi \in C_{0}^{\infty}(\bar{\Omega})$ there holds

$$
\begin{aligned}
\int_{\Omega_{k}}\left|\nabla v_{k}\right|^{N-2} \nabla v_{k} \nabla \varphi & =\int_{\Omega_{k}} \mu_{k}^{N-1}\left|\nabla u_{k}\right|^{N-2}\left(x_{k}+\mu_{k} x\right) \nabla u_{k}\left(x_{k}+\mu_{k} x\right) \nabla \varphi(x) d x \\
& =\mu_{k}^{N-1} \int_{\Omega}\left|\nabla u_{k}\right|^{N-2}(y) \nabla u_{k}(y)(\nabla \varphi)\left(\frac{y-x_{k}}{\mu_{k}}\right) \mu_{k} \frac{d y}{\mu_{k}^{N}} \\
& =\int_{\Omega} f\left(u_{k}(y)\right) \varphi\left(\frac{y-x_{k}}{\mu_{k}}\right) d y \\
& =\int_{\Omega_{k}} f\left(u_{k}\left(x_{k}+\mu_{k} x\right)\right) \varphi(x) \mu_{k}^{N} d x \\
& =\int_{\Omega_{k}} \frac{f\left(u_{k}\left(x_{k}+\mu_{k} x\right)\right)}{f\left(M_{k}\right)} \varphi(x) d x .
\end{aligned}
$$

Moreover, fixing $R>0$ and looking at the behaviour of the sequence $\left(v_{k}\right)_{k}$ in $B_{R}(0)$, applying the Harnack inequality given by Lemma 2.2 to $w_{k}:=-v_{k} \geq 0$, we find

$$
\left\|v_{k}\right\|_{L^{\infty}\left(B_{R}(0)\right)}=\max _{B_{R}(0)}\left|v_{k}\right|=\max _{B_{R}(0)} w_{k} \leq C(R)\left(\min _{B_{R}(0)} w_{k}+K(R)\right)=\tilde{K}(R) .
$$

Therefore, by the local estimate provided by Lemma 2.1, we infer that, up to a subsequence, $v_{k} \rightarrow v$ in $C_{l o c}^{1, \alpha}\left(\mathbb{R}^{N}\right)$. We claim that $v$ is a weak solutions of the Liouville's equation

$$
-\Delta_{N} v=e^{\beta v} \quad \text { in } \mathbb{R}^{N},
$$

where $\beta$ is defined in (A3). Indeed, the equation (10) can be rewritten as

$$
-\Delta_{N} v_{k}(x)=\exp \left\{\log \circ f\left(u_{k}\left(x_{k}+\mu_{k} x\right)\right)-\log \circ f\left(M_{k}\right)\right\}
$$

and thus, by a first-order Taylor expansion of $\log \circ f$ around $M_{k}$, one finds

$$
-\Delta_{N} v_{k}(x)=e^{\frac{f^{\prime}\left(z_{k}(x)\right)}{f\left(z_{k}(x)\right)}\left(u_{k}\left(x_{k}+\mu_{k} x\right)-M_{k}\right)}=e^{\frac{f^{\prime}\left(z_{k}(x)\right)}{f\left(z_{k}(x)\right)} v_{k}(x)},
$$

where $z_{k}(x):=M_{k}+\theta(x)\left(u_{k}\left(x_{k}+\mu_{k} x\right)-M_{k}\right)=M_{k}+\theta(x) v_{k}(x), \theta(x) \in(0,1)$. Since $v_{k} \rightarrow v$ uniformly on compact sets and $M_{k} \rightarrow+\infty$, then $z_{k}(x) \rightarrow+\infty$ uniformly on compact sets, so (12) follows by taking the limits as $k \rightarrow+\infty$ in (13).

Consequently, we split our analysis according to whether $f$ is subcritical or critical in the sense of Definition 1.1.

\subsection{The subcritical case $(\beta=0)$}

In this case the equation (12) satisfied by the limit function $v$ reduces to

$$
-\Delta_{N} v=1 \quad \text { in } \mathbb{R}^{N} \text {. }
$$

Recalling the energy estimate (6) of Proposition 2.3, it is sufficient to integrate (14) on $\mathbb{R}^{N}$ to get the desired contradiction, which proves Theorem 1.1 for such nonlinearities:

$$
\begin{aligned}
+\infty=\int_{\mathbb{R}^{N}} d x & =\int_{\mathbb{R}^{N}} \lim _{k \rightarrow+\infty} e^{\frac{f^{\prime}\left(z_{k}(x)\right)}{f\left(z_{k}(x)\right)} v_{k}(x)} \chi_{\Omega_{k}}(x) d x \\
& =\int_{\mathbb{R}^{N}} \lim _{k \rightarrow+\infty} e^{\log \left(f\left(u_{k}\left(x_{k}+\mu_{k} x\right)\right)\right)-\log \left(f\left(M_{k}\right)\right)} \chi_{\Omega_{k}}(x) d x \\
& \leq \liminf _{k \rightarrow+\infty} \int_{\Omega_{k}} \frac{f\left(u_{k}\left(x_{k}+\mu_{k} x\right)\right)}{f\left(M_{k}\right)} d x \\
& \stackrel{\left[y=x_{k}+\mu_{k} x\right]}{=} \liminf _{k \rightarrow+\infty} \int_{\Omega} f\left(u_{k}(y)\right) d y \leq \Lambda .
\end{aligned}
$$




\subsection{The critical case $(\beta>0)$}

With the same steps as in (15), one actually gets

$$
\int_{\mathbb{R}^{N}} e^{\beta v} d x \leq \Lambda
$$

Therefore, we can characterize the limit profile $v$ by standard computations from a Liouville-type result by Esposito [24, Theorem 1.1] as

$$
v(x)=-\frac{N}{\beta} \log \left(1+\left(\frac{\beta^{N-1}}{C_{N}}|x|^{N}\right)^{\frac{1}{N-1}}\right),
$$

where $C_{N}:=N\left(\frac{N^{2}}{N-1}\right)^{N-1}$. Furthermore, we claim that the energy concentrates around the blow-up points sequence $\left(x_{k}\right)_{k}$, namely there exists a constant $\theta>0$ such that

$$
\lim _{R \rightarrow+\infty} \liminf _{k \rightarrow+\infty} \int_{B_{R \mu_{k}}\left(x_{k}\right)} f\left(u_{k}(y)\right) d y \geq \theta>0,
$$

where $\theta$ is characterized by

$$
\theta=\frac{N \omega_{N}}{\beta^{N-1}}\left(\frac{N^{2}}{N-1}\right)^{N-1}
$$

with $\omega_{N}$ denoting the volume of the unit ball in $\mathbb{R}^{N}$. Indeed,

$$
\begin{aligned}
0<\theta: & =\int_{\mathbb{R}^{N}} e^{\beta v}=\lim _{R \rightarrow+\infty} \int_{B_{R}(0)} \lim _{k \rightarrow+\infty} e^{\left(\frac{f^{\prime}\left(z_{z(x))}\right.}{f\left(z_{k}(x)\right)}\right) v_{k}(x)} d x \\
& \leq \lim _{R \rightarrow+\infty} \liminf _{k \rightarrow+\infty} \int_{B_{R}(0)} \frac{f\left(u_{k}\left(x_{k}+\mu_{k} x\right)\right.}{f\left(M_{k}\right)} d x \\
& =\lim _{R \rightarrow+\infty} \liminf _{k \rightarrow+\infty} \int_{B_{R \mu_{k}}\left(x_{k}\right)} f\left(u_{k}(y)\right) d y .
\end{aligned}
$$

We can quantify the constant $\theta$ as follows. Let us define the auxiliary function $w:=\beta v+(N-1) \log \beta$. Then, it is easy to show that $w$ satisfies $-\Delta_{N} w=e^{w}$ and $\int_{\mathbb{R}^{N}} e^{w}<\infty$. Therefore, by [24, Theorem 1.1], we have

$$
\theta=\int_{\mathbb{R}^{N}} e^{\beta v}=\frac{1}{\beta^{N-1}} \int_{\mathbb{R}^{N}} e^{w}=\frac{N \omega_{N}}{\beta^{N-1}}\left(\frac{N^{2}}{N-1}\right)^{N-1}
$$

In other words, if we apply the blow-up technique around a maximum point, we see that we can characterize the limit profile and we get the concentration of the energy as in (18)-(19). This is indeed what happens around any blow-up point of the sequence $\left(u_{k}\right)_{k}$, meaning points belonging to the set

$$
S:=\left\{y \in \bar{\Omega}\left|\exists\left(y_{j}\right)_{j} \subseteq \Omega\right| y_{j} \rightarrow y, u_{k_{j}}\left(y_{j}\right) \rightarrow+\infty \text { as } j \rightarrow+\infty\right\} .
$$

Lemma 3.1. From the sequence $\left(u_{k}\right)_{k}$ one can extract a subsequence still denoted by $\left(u_{k}\right)_{k}$ for which the following holds.

There are $P \in \mathbb{N}$ and sequences $\left(x_{k, i}\right)_{k}$ for $1 \leq i \leq P$ such that $\lim _{k \rightarrow+\infty} u_{k}\left(x_{k, i}\right)=+\infty$ for any $i$ and, setting

$$
v_{k, i}(x):=u_{k}\left(x_{k, i}+\mu_{k, i} x\right)-u_{k}\left(x_{k, i}\right), \quad \mu_{k, i}:=\left(f\left(u_{k}\left(x_{k, i}\right)\right)\right)^{-1 / N},
$$

and $x^{(i)}=\lim _{k \rightarrow+\infty} x_{k, i}$, we have

(i) $\lim _{k \rightarrow+\infty} \frac{\left|x_{k, i}-x_{k, j}\right|}{\mu_{k, i}}=+\infty$ for $1 \leq i \neq j \leq P$;

(ii) $v_{k, i} \rightarrow v$ in $C_{l o c}^{1, \gamma}\left(\mathbb{R}^{N}\right)$, for $1 \leq i \leq P$, where $v$ is defined in (17) and (18)-(19) still hold;

(iii) $\sup _{k} \inf _{1 \leq i \leq P}\left|x-x_{k, i}\right|^{N} f\left(u_{k}(x)\right) \leq C$ for every $x \in \Omega$. 
Proof. The proof is inspired by [18, Claims 5-7]. We say that the property $\mathcal{H}_{p}$ holds whenever there exist $p$ sequences $\left(x_{k, i}\right)_{k}$ for $1 \leq i \leq p$ such that $u_{k}\left(x_{k, i}\right) \rightarrow+\infty$ as $k \rightarrow+\infty$ and such that (i) and (ii) hold. From our previous investigation, we know that $\mathcal{H}_{1}$ holds. Our aim is to prove that the number of such sequences is finite by showing the following: if $\mathcal{H}_{p}$ holds then either $\mathcal{H}_{p+1}$ holds too or there exists a constant $C>0$ such that (iii) holds. Indeed, if so, supposing by contradiction that $\mathcal{H}_{p}$ holds for any $p$, then we would be able to find a sequence of disjoint balls $B_{R \mu_{k, i}}\left(x_{k, i}\right)$ such that (18) holds, and thus by Lemma 2.3,

$$
\Lambda \geq \int_{\Omega} f\left(u_{k}(x)\right) d x \geq \int_{\bigcup_{i=1}^{p} B_{R \mu_{k, i}}\left(x_{k, i}\right)} f\left(u_{k}(x)\right) d x=\sum_{i=1}^{p} \int_{B_{R u_{k, i}}\left(x_{k, i}\right)} f\left(u_{k}(x)\right) d x \geq p \theta,
$$

an upper bound for $p$, a contradiction. As a consequence, $\mathcal{H}_{p}$ does not hold for any $p$ and, by the claimed alternative, the proof is completed.

Now the claim has to be proved. Suppose that $\mathcal{H}_{p}$ for some $p \in \mathbb{N}$ but not (iii). Hence, defining $w_{k}(x):=$ $\inf _{1 \leq i \leq p}\left|x-x_{k, i}\right|^{N} f\left(u_{k}(x)\right)$, one can find a sequence of points $\left(y_{k}\right)_{k}$ such that $w_{k}\left(y_{k}\right)=\left\|w_{k}\right\|_{\infty} \lambda+\infty$. We show that for these points $\left(y_{k}\right)_{k}$ together with $\left(x_{k, i}\right)_{k}$ for $1 \leq i \leq P, \mathcal{H}_{p+1}$ holds. First we get $u_{k}\left(y_{k}\right) \rightarrow+\infty$, since

$$
+\infty \leftarrow w_{k}\left(y_{k}\right) \leq(\operatorname{diam}(\Omega))^{N} f\left(u_{k}\left(y_{k}\right)\right)
$$

and by the local boundedness of $f$ on $[0,+\infty)$. Moreover, defining

$$
\tilde{u}_{k}(x):=u_{k}\left(y_{k}+\gamma_{k} x\right)-u_{k}\left(y_{k}\right), \quad \gamma_{k}:=\left(f\left(u_{k}\left(y_{k}\right)\right)\right)^{-1 / N},
$$

first we have

$$
\inf _{1 \leq i \leq p} \frac{\left|y_{k}-x_{k, i}\right|}{\gamma_{k}}=\inf _{1 \leq i \leq p}\left|y_{k}-x_{k, i}\right| f\left(u_{k}\left(y_{k}\right)\right)^{1 / N}=w_{k}\left(y_{k}\right)^{1 / N} \rightarrow+\infty .
$$

Then, suppose by contradiction that $\left|y_{k}-x_{k, i}\right|=O\left(\mu_{k, i}\right)$, that is $y_{k}=x_{k, i}+\mu_{k, i} \theta_{k, i}$, with $\left|\theta_{k, i}\right| \leq C$. We have

$$
\begin{aligned}
w_{k}\left(y_{k}\right) & \leq\left|y_{k}-x_{k, i}\right|^{N} f\left(u_{k}\left(y_{k}\right)\right)=\mu_{k, i}^{N}\left|\theta_{k, i}\right|^{N} f\left(u_{k}\left(y_{k}\right)\right)=\left|\theta_{k, i}\right|^{N} \frac{f\left(u_{k}\left(y_{k}\right)\right)}{f\left(u_{k}\left(x_{k, i}\right)\right)} \\
& =\left|\theta_{k, i}\right|^{N} e^{\frac{f^{\prime}\left(z_{k, i}\left(\theta_{k, i}\right)\right)}{f\left(z_{z, i}\left(\theta_{k, i}\right)\right.} v_{k, i}\left(\theta_{k, i}\right)} \rightarrow\left|\theta_{\infty, i}\right|^{N} e^{\beta v\left(\theta_{\infty, i}\right)}=\frac{\left|\theta_{\infty, i}\right|^{N}}{\left(1+\beta C_{N} \frac{1}{1-N}\left|\theta_{\infty, i}\right|^{\frac{N}{N-1}}\right)^{N}}<+\infty,
\end{aligned}
$$

a contradiction, so $(i)$ is proved.

Now, it remains to prove that $u_{k}$ has the same blow-up behaviour also for the sequence $\left(y_{k}\right)_{k}$. To this aim, we first show that, for any $R>0$

$$
\frac{f\left(u_{k}\left(y_{k}+\gamma_{k} x\right)\right)}{f\left(u_{k}\left(y_{k}\right)\right)} \leq C
$$

for some $C=C(R)>0$ and for any $x \in B_{R}(0)$. Notice that this is not obvious as in (10), since $\left(y_{k}\right)_{k}$ do not have to be necessarily maximum points for $\left(u_{k}\right)_{k}$. Rewriting in terms of the sequence $\left(u_{k}\right)_{k}$ the inequality $w_{k}\left(y_{k}+\gamma_{k} x\right) \leq w_{k}\left(y_{k}\right)$, which holds for any $x \in B_{R}(0)$ for $k$ large enough, one finds

$$
\frac{f\left(u_{k}\left(y_{k}+\gamma_{k} x\right)\right)}{f\left(u_{k}\left(y_{k}\right)\right)} \leq\left(\frac{\inf _{1 \leq i \leq p}\left|y_{k}-x_{k, i}\right|}{\inf _{1 \leq i \leq p}\left|y_{k}+\gamma_{k} x-x_{k, i}\right|}\right)^{N} \text {. }
$$

Fix now $\varepsilon \in(0,1)$. By (20), there exists $\bar{k}=\bar{k}(R, \varepsilon)>0$ such that for any $k>\bar{k}$ we have $R \gamma_{k} \leq \varepsilon\left|y_{k}-x_{k, i}\right|$. Therefore $\left|y_{k}+\gamma_{k} x-x_{k, i}\right| \geq\left|y_{k}-x_{k, i}\right|-\gamma_{k} R \geq(1-\varepsilon)\left|y_{k}-x_{k, i}\right|$ and so (21) follows from (22) with $C=(1-\varepsilon)^{-N}$. In order to complete the proof of the local compactness of the sequence $\left(\tilde{u}_{k}\right)_{k}$ and consequently get (ii), we need to have its local boundedness in the $L^{N}$ norm according to Lemma 2.1. This time, unlike (11), before applying the Harnack inequality of Lemma 2.2, we need to know that our sequence is uniformly bounded from above, which is not immediate. So, suppose by contradiction that for any sequence of positive constants $\left(C_{k}\right)_{k} \lambda+\infty$ there exist points $\left(\tilde{x}_{k}\right)_{k} \subset B_{R}(0)$ such that $\tilde{u}_{k}\left(\tilde{x}_{k}\right) \geq C_{k}$ holds, thus, since $f$ is increasing,

$$
f\left(u_{k}\left(y_{k}+\gamma_{k} \tilde{x}_{k}\right)\right) \geq f\left(C_{k}+u_{k}\left(y_{k}\right)\right) .
$$


Together with (21), this implies

$$
f\left(C_{k}+u_{k}\left(y_{k}\right)\right) \leq C f\left(u_{k}\left(y_{k}\right)\right) .
$$

Choosing now $C_{k}=e^{u_{k}\left(y_{k}\right)^{2}}$ and setting $t_{k}:=u_{k}\left(y_{k}\right)$, (23) rereads as

$$
f\left(e^{t_{k}^{2}}+t_{k}\right) \leq C f\left(t_{k}\right) .
$$

Then, by superlinearity of $f$ and by the fact that $f(s) \leq e^{\gamma s}+D$ for some $\gamma, D>0$ for any $s \in \mathbb{R}^{+}$by (A3), we have

$$
e^{t_{k}^{2}}+t_{k}-\bar{C} \leq f\left(e^{t_{k}^{2}}+t_{k}\right) \leq C f\left(t_{k}\right) \leq e^{\gamma t_{k}}+D
$$

which yields a contradiction since $t_{k} \lambda+\infty$. Hence, $M(R):=\max _{B_{R}(0)} \tilde{u}_{k} \in[0,+\infty)$ and we can consider the function $U_{k}:=M(R)-\tilde{u}_{k}$. We have $U_{k} \geq 0, \min _{B_{R}(0)} U_{k}=0$ and furthermore $U_{k}$ satisfies

$$
-\Delta_{N} U_{k}=-\frac{f\left(u_{k}\left(y_{k}+\gamma_{k} x\right)\right)}{f\left(u_{k}\left(y_{k}\right)\right)} \in[-C(R), 0],
$$

where the bound from below follows from (21). Therefore, by Lemma 2.2, we get

$$
\left|M(R)+\min _{B_{R}(0)} \tilde{u}_{k}\right|=\max _{B_{R}(0)}\left|U_{k}\right|=\max _{B_{R}(0)} U_{k} \leq C(R)\left[\min _{B_{R}(0)} U_{k}+K(R)\right]=C(R) K(R),
$$

which in turn implies

$$
\left|\max _{B_{R}(0)} \tilde{u}_{k}-\right| \min _{B_{R}(0)} \tilde{u}_{k}|| \leq \tilde{C}(R) .
$$

Therefore, either $\max _{B_{R}(0)} \tilde{u}_{k} \geq\left|\min _{B_{R}(0)} \tilde{u}_{k}\right|$, and in this case we easily infer $\left\|\tilde{u}_{k}\right\|_{L^{\infty}\left(B_{R}(0)\right)} \leq C(R)$, or we deduce from (25) that

$$
\left|\min _{B_{R}(0)} \tilde{u}_{k}\right|-\max _{B_{R}(0)} \tilde{u}_{k} \leq \tilde{C}(R) .
$$

This readily implies $\min _{B_{R}(0)} \tilde{u}_{k} \geq-C(R)$, so again we find $\left\|\tilde{u}_{k}\right\|_{L^{\infty}\left(B_{R}(0)\right)} \leq C(R)$.

Applying Lemma 2.1, we infer that $\left(\tilde{u}_{k}\right)_{k}$ is locally compact in $C_{l o c}^{1, \alpha}\left(\mathbb{R}^{N}\right)$ and, with similar steps as in (13)-(17), we finally infer (ii). Consequently, the property $\mathcal{H}_{p}$ is satisfied and the proof is concluded.

Corollary 3.2. The set of blow-up points is finite.

Proof. We show the finiteness of $S$ by proving that $S=\left\{x^{(i)}, 1 \leq i \leq P\right\}$, where the points $x^{(i)}$ are defined as in Lemma 3.1. Indeed, let $\bar{x} \notin\left\{x^{(i)}, 1 \leq i \leq P\right\}$ for which one can find a sequence $\bar{x}_{k} \rightarrow \bar{x}$ such that, up to a subsequence, $u_{k}\left(\bar{x}_{k}\right) \rightarrow+\infty$. We have $\inf _{1 \leq i \leq P, k \in \mathbb{N}}\left|\bar{x}_{k}-x^{(i)}\right| \geq \bar{d}>0$ and, by Proposition $2.3, d\left(\bar{x}_{k}, \partial \Omega\right) \geq \eta>0$ for some constants $\bar{d}, \eta$. Then, by (iii) of Lemma 3.1 and the superlinearity of $f$, we infer

$$
C \geq \inf _{1 \leq i \leq P}\left|\bar{x}_{k}-x_{k, i}\right|^{N} f\left(u_{k}\left(\bar{x}_{k}\right)\right) \geq \frac{\bar{d}^{N}}{2}\left(u_{k}\left(\bar{x}_{k}\right)-C\right),
$$

a contradiction.

Now we are in the position to prove Theorem 1.1.

Proof of Theorem 1.1. By (7) $S$ is nonempty. Let $x_{0} \in S$ and $r$ small such that $S \cap B_{r}\left(x_{0}\right)=\left\{x_{0}\right\}$. In this way, $\left\|u_{k}\right\|_{L^{\infty}(K)}<C$ for any $K \subset B_{r}\left(x_{0}\right) \backslash\left\{x_{0}\right\}$ and thus $u_{k} \rightarrow \tilde{u}$ in $C_{l o c}^{1, \alpha}\left(B_{r}\left(x_{0}\right) \backslash\left\{x_{0}\right\}\right)$ by Lemma 2.1. Moreover, by the energy bound of Proposition 2.3, the sequence $\left(f_{k}\right)_{k}:=\left(f\left(u_{k}\right)\right)_{k}$ is bounded in $L^{1}$, thus $f_{k} \rightarrow \mu$ in $\mathcal{M}\left(B_{r}\left(x_{0}\right)\right) \cap L_{\text {loc }}^{\infty}\left(B_{r}\left(x_{0}\right) \backslash\left\{x_{0}\right\}\right)$ where $\mu$ is a positive measure which is singular at $x_{0}$. Hence, we can decompose $\mu$ as

$$
\mu=A(x) d x+a_{0} \delta_{x_{0}},
$$

where $0 \leq A \in L^{1}\left(B_{r}\left(x_{0}\right)\right), a_{0}$ is a positive constant and $\delta_{x_{0}}$ denotes the Dirac distribution centered at $x_{0}$. We first claim that $a_{0} \geq \theta$ with $\theta$ as in (18)-(19). Indeed, for any $t \in(0, r)$ we have

$$
\int_{B_{t}\left(x_{0}\right)} d \mu=\lim _{k \rightarrow+\infty} \int_{B_{t}\left(x_{0}\right)} f\left(u_{k}(x)\right) d x \geq \liminf _{k \rightarrow+\infty} \int_{B_{R \mu_{k}}\left(x_{k}\right)} f\left(u_{k}(x)\right) d x \geq \theta
$$


by (18), where here $x_{k}$ is a blow-up sequence converging to $x_{0}$. As $t$ is arbitrary, we thus find $a_{0} \geq \theta$.

Let now $w$ be the distributional solution of

$$
\begin{cases}-\Delta_{N} w=a_{0} \delta_{x_{0}} & \text { in } B_{r}\left(x_{0}\right), \\ w=0 & \text { on } \partial B_{r}\left(x_{0}\right) .\end{cases}
$$

Then by [25, Theorem 2.1] $w \in C^{1, \alpha}\left(B_{r}\left(x_{0}\right) \backslash\left\{x_{0}\right\}\right)$ and has the explicit form

$$
w(x)=\left(\frac{a_{0}}{N \omega_{N}}\right)^{\frac{1}{N-1}} \log \left(\frac{r}{\left|x-x_{0}\right|}\right) .
$$

We claim that

$$
w \leq \tilde{u} \quad \text { in } B_{r}\left(x_{0}\right) .
$$

If so, choosing $\varepsilon \in\left(0, \frac{\beta}{N}\right)$, in view of (2) on the one hand by Proposition 2.3 we get

$$
\begin{aligned}
\int_{B_{r}\left(x_{0}\right)} e^{(\beta-\varepsilon) w} & \leq \int_{B_{r}\left(x_{0}\right)} e^{(\beta-\varepsilon) \tilde{u}} \leq \liminf _{k \rightarrow+\infty} \int_{B_{r}\left(x_{0}\right)} e^{(\beta-\varepsilon) u_{k}} \\
& \leq c \liminf _{k \rightarrow+\infty} \int_{B_{r}\left(x_{0}\right)} f\left(u_{k}\right)+d \leq C(\Lambda) .
\end{aligned}
$$

On the other hand, by means of the explicit expressions for $w$ and $\theta$ :

$$
\begin{aligned}
\int_{B_{r}\left(x_{0}\right)} e^{(\beta-\varepsilon) w} & =\int_{B_{r}\left(x_{0}\right)}\left(\frac{r}{\left|x-x_{0}\right|}\right)^{(\beta-\varepsilon)\left(\frac{a_{0}}{N \omega_{N}}\right)^{\frac{1}{N-1}}} d x \\
& \geq \int_{B_{r}\left(x_{0}\right)}\left(\frac{r}{\left|x-x_{0}\right|}\right)^{(\beta-\varepsilon)\left(\frac{\theta}{N \omega_{N}}\right)^{\frac{1}{N-1}}} d x \\
& \geq \int_{B_{r}\left(x_{0}\right)}\left(\frac{r}{\left|x-x_{0}\right|}\right)^{\frac{(\beta-\varepsilon)}{\beta}\left(\frac{N^{2}}{N-1}\right)} d x \\
& >\int_{B_{r}\left(x_{0}\right)}\left(\frac{r}{\left|x-x_{0}\right|}\right)^{N} d x=+\infty,
\end{aligned}
$$

a contradiction. Therefore the proof of Theorem 1.1 is completed once we prove (29). Here, we follow the strategy in [1]. For any $j \in \mathbb{N}$, let us define the maps $B_{j}: \overline{\mathbb{R}} \rightarrow \mathbb{R}^{+}$by

$$
B_{j}(s)= \begin{cases}0 & \text { for } s<0 \\ s & \text { for } 0 \leq s \leq j, \\ j & \text { for } s>j\end{cases}
$$

and the functions $z_{k}^{(j)}:=B_{j}\left(w-u_{k}\right) \geq 0$. Then, it is easy to see that $z_{k}^{(j)}\left(x_{0}\right)=j$ for any $k$, that $\left.z_{k}^{(j)}\right|_{\partial B_{r}\left(x_{0}\right)}=0$ and, moreover, $z_{k}^{(j)} \in W_{0}^{1, N}\left(B_{R}\left(x_{0}\right)\right)$. Furthermore, as $B_{j}$ is continuous, one has $z_{k}^{(j)} \rightarrow z^{(j)}$ pointwise, where

$$
z^{(j)}(s)= \begin{cases}B_{j}(w-\tilde{u}) & \text { for } x \neq x_{0} \\ j & \text { for } x=x_{0}\end{cases}
$$

Notice that $z_{k}^{(j)}$ may be extended to 0 in $\Omega \backslash B_{r}\left(x_{0}\right)$. Since $w$ and $u_{k}$ solve respectively problems (28) and (3), then

$$
\begin{aligned}
\int_{B_{r}\left(x_{0}\right)}\left(|\nabla w|^{N-2} \nabla w-|\nabla \tilde{u}|^{N-2} \nabla \tilde{u}\right) \nabla z_{k}^{(j)} & =a_{0} z_{k}^{(j)}\left(x_{0}\right)-\int_{\Omega} f\left(u_{k}\right) z_{k}^{(j)} \\
& =a_{0} j-\int_{B_{r}\left(x_{0}\right)} f\left(u_{k}\right) z_{k}^{(j)}
\end{aligned}
$$


Recalling $f\left(u_{k}\right) \rightarrow \mu$ and (26), using the generalized Fatou's Lemma with measures (see e.g. [26, Chapter 11, Proposition 17]), one infers

$$
\liminf _{k \rightarrow+\infty} \int_{B_{r}\left(x_{0}\right)} f\left(u_{k}\right) z_{k}^{(j)} d x \geq \int_{B_{r}\left(x_{0}\right)} z^{(j)} d \mu \geq \int_{\left\{x_{0}\right\}} z^{(j)} d \mu \geq a_{0} j,
$$

and thus

$$
\int_{B_{r}\left(x_{0}\right)}\left(|\nabla w|^{N-2} \nabla w-|\nabla \tilde{u}|^{N-2} \nabla \tilde{u}\right) \nabla z^{(j)} \leq 0
$$

that is,

$$
\int_{B_{r}\left(x_{0}\right) \cap\{0 \leq w-\tilde{u} \leq j\}}\left(|\nabla w|^{N-2} \nabla w-|\nabla \tilde{u}|^{N-2} \nabla \tilde{u}\right) \nabla(w-\tilde{u}) \leq 0,
$$

which holds for any choice of $j \in \mathbb{N}$. Then, the well-known inequality

$$
d_{N}|X-Y|^{N} \leq\left\langle|X|^{N-2} X-|Y|^{N-2} Y, X-Y\right\rangle,
$$

for any $X \neq Y \in \mathbb{R}^{N}$, the constant $d_{N}>0$ depending only on $N$ (see [27, Proposition 4.6]), implies

$$
d_{N} \int_{B_{r}\left(x_{0}\right)}\left|\nabla(w-\tilde{u})^{+}\right|^{N} \leq 0
$$

which finally proves our claim $w-\tilde{u} \leq 0$ in $B_{r}\left(x_{0}\right)$, since $(w-\tilde{u})^{+} \leq 0$ on $\partial B_{r}\left(x_{0}\right)$.

\section{Generalization to nonhomogeneous problems}

So far, we studied the homogeneous quasilinear problem (3), which allows a clearer exposition and a more direct comparison with the results in [1, 2]. However, a similar analysis can be carried out also in the nonhomogeneous setting, provided some conditions at infinity and near the boundary are fulfilled. More precisely, we may consider the problem

$$
\begin{cases}-\Delta_{N} u=h(x, u) & \text { in } \Omega, \\ u=0 & \text { on } \partial \Omega,\end{cases}
$$

where $h: \bar{\Omega} \times \mathbb{R} \rightarrow \mathbb{R}^{+} \cup\{0\}$ is a Carathéodory function satisfying the following conditions:

H1) $h \in L^{\infty}(\Omega \times[0, \tau])$ for all $\tau \in \mathbb{R}^{+}$and $h(x, t)>0$ for any $x \in \Omega$ and $t>0$;

H2) there exist $f \in C^{1}([0,+\infty))$ satisfying $(A 1)-(A 3)$ and $0<a \in L^{\infty}(\Omega) \cap C(\Omega)$ such that

$$
\lim _{t \rightarrow+\infty} \frac{h(x, t)}{a(x) f(t)}=1 \quad \text { uniformly in } \Omega ;
$$

H3) there exist $\bar{r}, \bar{\delta}>0$ such that

- $h(\cdot, t) \in C^{1}\left(\Omega_{\bar{r}}\right)$ for all $t \geq 0$ and $\frac{\partial h}{\partial t}(x, t) \geq 0$ in $\Omega_{\bar{r}} \times \mathbb{R}^{+}$;

- $\nabla_{x} h(x, t) \cdot \Psi \leq 0$ for all $x \in \Omega_{r}, t \geq 0$ and unit vectors $\Psi$ such that $|\Psi-n(x)| \leq \bar{\delta}$.

We recall $\Omega_{r}:=\{x \in \Omega \mid d(x, \partial \Omega) \leq r\}$.

Remark 4. In (H2) we assume $a>0$ only in the interior of $\Omega$, but it may vanish on the boundary. Moreover, assumption (H3) is imposed to uniformly control solutions near the boundary by a moving-planes technique, prescribing in broad terms that $h$ should be decreasing in $x$ along suitable outer directions and increasing in $t$ in a suitable neighbourhood of $\partial \Omega$.

Remark 5. From (H1)-(H2) it follows that for each $\varepsilon>0$ there exists a constant $d_{\varepsilon} \geq 0$ such that

$$
(1-\varepsilon) a(x) f(t)-d_{\varepsilon} \leq h(x, t) \leq(1+\varepsilon) a(x) f(t)+d_{\varepsilon} \quad \text { for all } t \geq 0, x \in \Omega .
$$


Theorem 4.1. Let $\Omega \subset \mathbb{R}^{N}, N \geq 2$, be a bounded strictly convex domain of class $C^{1, \alpha}$ for some $\alpha \in(0,1)$ and let $h$ satisfy (H1)-(H3). Then, there exists a constant $C>0$ such that

$$
\|u\|_{L^{\infty}(\Omega)} \leq C
$$

for all weak solutions $u \in W_{0}^{1, N}(\Omega)$ of problem (3).

The proof of Theorem 4.1 mainly follows the arguments of the previous sections, so we sketch here only the remarkable modifications.

Proof. First, we want to prevent blow-up near the boundary, so we adapt in our context the results of Proposition 2.3. With the same argument as in [1, Proposition 2.1] and taking (31) into account, one infers that solutions are bounded in $L_{l o c}^{d}(\Omega)$, namely

$$
\int_{\Omega} u^{d} \Phi_{1}^{N} \leq C
$$

where $d$ is defined in (A2), $C$ is independent of $u$ and $\Phi_{1} \in W_{0}^{1, N}(\Omega)$ is the first (positive) eigenfunction of $-\Delta_{N}$ on $\Omega$. In order to prove that (32) yields a uniform bound near $\partial \Omega$, we have to show that here our solutions are decreasing with respect to outer directions. We apply a moving-planes technique in the spirit of [28, Lemma 3.2]. Let us first fix some notation. For any direction $v$, set

$$
T_{\lambda}^{v}:=\left\{x \in \mathbb{R}^{N} \mid x \cdot v=\lambda\right\} \quad \text { and } \quad \Omega_{\lambda}^{v}:=\{x \in \Omega \mid x \cdot v<\lambda\},
$$

the latter being nonempty for $\lambda>a(v):=\inf _{x \in \Omega} x \cdot v$. Moreover, for any $x \in \Omega$, we denote by $x_{\lambda}^{v}$ the symmetric point with respect to the hyperplane $T_{\lambda}^{v}$, namely

$$
x_{\lambda}^{v}=R_{\lambda}^{v}(x):=x+2(\lambda-x \cdot v) \nu .
$$

Similarly, we define $u_{\lambda}^{v}:=R_{\lambda}^{v}(u)$, which is well-defined on $\left(\Omega_{\lambda}^{v}\right)^{\prime}:=R_{\lambda}^{v}\left(\Omega_{\lambda}^{v}\right)$. Our aim is to compare $u$ and $u_{\lambda}^{v}$ near the boundary in the case $v=n(x)$ (the outer normal) and $\lambda-a(v)$ small, in order to conclude that $u$ is decreasing along these directions in a small neighborhood of $\partial \Omega$. First notice that by convexity of $\Omega$, as long as $\lambda-a(v)$ is small, we have $\left(\Omega_{\lambda}^{v}\right)^{\prime} \subset \Omega$ and $\Omega_{\lambda}^{v} \cup\left(\Omega_{\lambda}^{v}\right)^{\prime} \subset \Omega_{\bar{r}}$. Therefore, for such $\lambda$, in $\Omega_{\lambda}^{v}$ one has

$$
\begin{aligned}
-\Delta_{N} u_{\lambda}^{v}(x)-\left(-\Delta_{N} u(x)\right) & =h\left(x_{\lambda}^{v}, u_{\lambda}^{v}\right)-h(x, u) \stackrel{(H 3)}{\geq} h\left(x, u_{\lambda}^{v}\right)-h(x, u) \\
& =M(x)\left(u_{\lambda}^{v}-u\right),
\end{aligned}
$$

where

$$
M(x)=\frac{\partial h}{\partial t}(x, \eta(x, \lambda) \stackrel{(H 3)}{\geq} 0
$$

for some real number $\eta(x, \lambda)$ lying between $u_{\lambda}^{v}(x)$ and $u(x)$ by the mean value theorem, hence $M \in L^{\infty}\left(\Omega_{\lambda}^{v}\right)$. Therefore, $u_{\lambda}^{v}$ et $u$ satisfy

$$
\begin{cases}-\Delta_{N} u_{\lambda}^{v}-M(x) u_{\lambda}^{v} \geq-\Delta_{N} u-M(x) u & \text { in } \Omega_{\lambda}^{v}, \\ u_{\lambda}^{v} \geq u & \text { on } \partial \Omega_{\lambda}^{v},\end{cases}
$$

and the weak comparison principle in small domains [12, Theorem 1.3] yields $u_{\lambda}^{v} \geq u$ in $\Omega_{\lambda}^{v}$. We point out that the mentioned result is originally stated for $M$ positive constant and for homogeneous nonlinearities, but it can be easily adapted to our setting (here assumption (H1) is required). By arbitrariness of $\lambda$ and $x \in \Omega_{\bar{r}}$, one deduces that $u$ is decreasing along the outer normal direction and then, by a compactness argument, that there exist $\delta \in(0, \bar{\delta}]$ and $r \in(0, \bar{r}]$ independent of $u$ such that $\nabla u(x) \cdot \Psi \leq 0$ for every $x \in \Omega_{r}$ and for every direction $\Psi$ such that $|\Psi-n(x)|<\delta$. The boundedness of $u$ and $\nabla u$ in a neighbourhood of the boundary now follows by a standard argument by [5], and moreover one infers

$$
\int_{\Omega} h(x, u) d x \leq \Lambda,
$$

with $\Lambda$ independent of $u$. We refer to [1, Propositions 2.1-3.1] for the details. 
Let us now address to the blow-up analysis. We shall see that the argument carried out in Section 3 applies to the problem (30) with only minor modifications. In particular, the rescaled functions $v_{k}$ defined in (8)-(9) turn out to be weak solutions of

$$
-\Delta_{N} v_{k}(x)=\frac{h\left(x_{k}+\mu_{k} x, u_{k}\left(x_{k}+\mu_{k} x\right)\right)}{f\left(M_{k}\right)}, \quad x \in \Omega_{k}:=\frac{\Omega-x_{k}}{\mu_{k}},
$$

and one shows that $v_{k} \rightarrow v$ in $C_{\text {loc }}^{1, \alpha}\left(\mathbb{R}^{N}\right)$ which satisfies

$$
-\Delta_{N} V=a\left(x_{\infty}\right) e^{\beta v} \quad \text { in } \mathbb{R}^{N} .
$$

We recall that $d\left(x_{\infty}, \partial \Omega\right)>0$, as we have already excluded boundary blow-up, thus $a\left(x_{\infty}\right)>0$ by (H2).

In the subcritical case, the same argument as in Section 3.1 holds and, in the critical framework, Lemma 3.1 easily adapts, showing that near blow-up points the energy concentrates:

$$
\lim _{R \rightarrow+\infty} \liminf _{k \rightarrow+\infty} \int_{B_{R \mu_{k}}\left(x_{k}\right)} h\left(y, u_{k}(y)\right) d y \geq \theta>0,
$$

with the same constant $\theta$ defined in (19). Then, the conclusion of the proof is analogous to the one for the homogeneous case.

\section{A counterexample}

As briefly mentioned in the Introduction, our assumption (A3) can be interpreted as a control from above for the growth at $\infty$ of the nonlinearity $f$ by a suitable power of $e^{t}$. In the spirit of Brezis-Merle [14, Example 2], we show an example of nonlinearities which are still subcritical in the sense of the Trudinger-Moser inequality without satisfying (A3) and for which one may find a positive potential $a(x)$ such that the problem (30) admits unbounded solutions, in a suitable distributional sense.

To this aim, let $f(t)=e^{t^{\alpha}}$ with $\alpha \in\left(1, \frac{N}{N-1}\right)$. Notice that $f$ clearly satisfies (A1) and (A2), it is subcritical in the sense of Trudinger-Moser, but the condition $(A 3)$ is not fulfilled. Moreover, fix parameters $\delta:=\frac{(\alpha-1) N+1}{\alpha N}>0$ and $\gamma:=\frac{\alpha-1}{\alpha} \in(0,1)$ and consider the family of functions

$$
\phi_{\beta}(t)=t+\beta t^{\gamma}-\delta \log t,
$$

where $\beta>0$ is a positive parameter that will be chosen later. Notice that $\phi_{\beta}(t)>t-\delta \log t$ and $\phi_{\beta}^{\prime}(t)>1-\frac{d}{t}$. Hence, defining

$$
u_{\beta}(r):=\phi_{\beta}(l(r))^{\frac{1}{\alpha}}, \quad r=|x|,
$$

where $l(r):=\log \left(\frac{1}{r}\right)$, there exists $\rho_{0}>0$ such that for any $r \in\left(0, \rho_{0}\right)$ there holds $u_{\beta}(r)>0$ and $u_{\beta}^{\prime}(r)<0$. In the sequel, we adapt the strategy in $[19, \S 6]$.

First, using an implicit function argument as in [19, Lemma 6.2], one can show (up to a smaller value of $\rho_{0}$ ) that for any $\rho \in\left(0, \rho_{0}\right)$ there exists $\beta=\beta(\rho) \sim l(\rho)^{\frac{1}{\alpha}}$ as $\rho \rightarrow 0$ such that $u_{\beta(\rho)}(\rho)-\frac{\beta(\rho)}{\alpha}=0$ on $\partial B_{\rho}(0)$. With this choice, one has $u_{\beta(\rho)}(r) \sim l(r)^{\frac{1}{\alpha}}$ as $r \rightarrow 0$. Then, for $r \in(0, \rho)$ we compute

$$
\begin{aligned}
-\Delta_{N} u_{\beta(\rho)}(r) & =-\frac{d}{d r}\left[r^{N-1}\left|u_{\beta(\rho)}^{\prime}(r)\right|^{N-2} u_{\beta(\rho)}^{\prime}(r)\right] \\
& =\frac{N-1}{\alpha^{N} r^{N}}\left[\left(1-\frac{1}{\alpha}\right) u_{\beta(\rho)}^{N-1-\alpha N}(r) \phi_{\beta(\rho)}^{\prime}(l(r))^{N}-u_{\beta(\rho)}^{(1-\alpha)(N-1)}(r) \phi_{\beta(\rho)}^{\prime}(l(r))^{N-2} \phi_{\beta(\rho)}^{\prime \prime}(l(r))\right] .
\end{aligned}
$$

Using the relations $\frac{1}{2} \leq \phi_{\beta(\rho)}^{\prime} \leq 1$ and $\phi_{\beta(\rho)}^{\prime \prime} \leq \delta l(r)^{-2}$ for $r$ sufficiently small, one infers from (33) that there exists $\rho_{1}<\rho_{0}$ such that for any $\rho \in\left(0, \rho_{1}\right)$ and $r \in(0, \rho)$ one has $-\Delta_{N} u_{\beta(\rho)}(r)>0$.

Let us now fix $\rho \in\left(0, \rho_{1}\right)$ and define

$$
w(r):=N^{\frac{1}{\alpha}}\left(u_{\beta(\rho)}(r)-\frac{\beta(\rho)}{\alpha}\right) .
$$


We have that $w>0$ solves pointwise

$$
\begin{cases}-\Delta_{N} w=a(x) e^{w^{\alpha}} & \text { in } B_{\rho}(0) \backslash\{0\} \\ w=0 & \text { on } \partial B_{\rho}(0)\end{cases}
$$

with $a(x):=-e^{-w^{\alpha}} \Delta_{N} w>0$. Now we claim that $a \in L^{\infty}\left(B_{\rho}(0)\right)$ but $w \notin L^{\infty}\left(B_{\rho}(0)\right)$. Indeed,

$$
\begin{aligned}
w^{\alpha}(r) & =N u_{\beta(\rho)}^{\alpha}(r)\left(1-\frac{\beta(\rho)}{\alpha u_{\beta(\rho)}(r)}\right)^{\alpha}=N u_{\beta(\rho)}^{\alpha}(r)-N \beta(\rho) u_{\beta(\rho)}^{\alpha-1}(r)+o(1) \\
& =N l(r)+N \beta(\rho) l(r)^{\gamma}-N \delta \log l(r)-N \beta(\rho) l(r)^{\frac{\alpha-1}{\alpha}}+o(1)
\end{aligned}
$$

as $r \rightarrow 0$. Recalling now that $\gamma=\frac{\alpha-1}{\alpha}$, we get

$$
w^{\alpha}(r)=N l(r)-N \delta \log l(r)+o(1)
$$

as $r \rightarrow 0$. Therefore,

$$
\begin{aligned}
a(x)=-N^{\frac{1}{\alpha}} \Delta_{N} u_{\beta(\rho)} e^{-w^{\alpha}} & =\frac{N^{\frac{1}{\alpha}}(N-1)(\alpha-1)}{\alpha^{N+1} r^{N}} l(r)^{\frac{N-1-\alpha N}{\alpha}} r^{N} l(r)^{N \delta}(1+o(1)) \\
& =\frac{N^{\frac{1}{\alpha}}(N-1)(\alpha-1)}{\alpha^{N+1}}+o(1) .
\end{aligned}
$$

as $r \rightarrow 0$, by our choice of $\delta$. Hence $a \in L^{\infty}\left(B_{\rho}(0)\right)$. However, $w$ is not bounded near 0 as it inherits the same behaviour of $u_{\beta(\rho)}$.

Now we want to prove that $w$ is an entropy solution of the problem (30). This kind of solutions has been introduced in the context of quasilinear elliptic problems in [29] in order to weaken the notion of weak solution for problems with $L^{1}$-data. Here we recall the precise meaning.

Definition 5.1. We say that $u \in T_{0}^{1, N}(\Omega)$ if $u$ is measurable and $T_{k} u \in W_{0}^{1, N}(\Omega)$ for any $k>0$, where $T_{k} u$ is defined as the truncation of $u$ at level $k$, namely

$$
T_{k}(s)= \begin{cases}-k & \text { for } s<-k, \\ s & \text { for }-k \leq s \leq k, \\ k & \text { for } s>k\end{cases}
$$

Definition 5.2. A function $u \in T_{0}^{1, N}(\Omega)$ is called an entropy solution of problem (30) if for any test function $\varphi \in C_{0}^{\infty}(\Omega)$ there holds

$$
\int_{\{|u-\varphi|<k\}}|\nabla u|^{N-2} \nabla u(\nabla u-\nabla \varphi) d x \leq \int_{\Omega} T_{k}(u-\varphi) h(x, u(x)) d x .
$$

We start proving $T_{k} w \in W_{0}^{1, N}\left(B_{\rho}(0)\right)$ for any $k>0$ and $w$ defined in (34). Indeed, $T_{k} w$ is radial and

$$
T_{k} w(r)= \begin{cases}w(r) & \text { for } r \in\left[r_{k}, \rho\right], \\ w\left(r_{k}\right) & \text { for } r \in\left[0, r_{k}\right),\end{cases}
$$

for some suitable $r_{k} \in(0, \rho)$, so it is easy to see that

$$
\int_{r_{k}}^{\rho}\left|\frac{d w}{d r}\right| r^{N-1} d r \sim \int_{r_{k}}^{\rho} w(r)^{N(1-\alpha)} \frac{(N-\gamma(1-\log r))^{N}}{r} d r<C_{k} .
$$

In order to show that $u$ satisfies (35), let $\varphi \in C_{0}^{\infty}\left(B_{\rho}(0)\right)$ and notice that $T_{k}(w-\varphi) \in W_{0}^{1, N}\left(B_{\rho}(0)\right)$. By our choice of $a$, the problem (30) is pointwise satisfied, thus we may test it in $B_{\rho}(0)$ by $T_{k}(w-\varphi)$ :

$$
-\int_{B_{\rho}(0)} \Delta_{N} w T_{k}(w-\varphi) d x=\int_{B_{\rho}(0)} a(x) e^{w^{\alpha}} T_{k}(w-\varphi) d x .
$$


Note that the right-hand side is well-defined as we proved that $a \in L^{\infty}\left(B_{\rho}(0)\right)$ and $e^{w^{\alpha}} \in L^{1}\left(B_{\rho}(0)\right)$. Integrating by parts the left-hand side and recalling $T_{k}(w-\varphi)_{\left.\right|_{\partial B_{\rho}(0)}}=0$, we have

$$
\int_{B_{\rho}(0)}|\nabla w|^{N-2} \nabla w \nabla T_{k}(w-\varphi) d x=\int_{B_{\rho}(0)} a(x) e^{w^{\alpha}} T_{k}(w-\varphi) d x
$$

which is the case of the equality in (35).

We have therefore showed that, although $w$ is an entropy solution of the problem

$$
\begin{cases}-\Delta_{N} w=a(x) e^{w^{\alpha}} & \text { in } B_{\rho}(0), \\ w=0 & \text { on } \partial B_{\rho}(0),\end{cases}
$$

however, $w \notin L^{\infty}\left(B_{\rho}(0)\right)$, as $w(r) \sim l(r)^{\frac{1}{\alpha}}$ as $r \rightarrow 0$.

Remark 6. This counterexample shows that the class of the nonlinearities considered by assumption (A3) is sharp in order to have the property of (uniform) boundedness within the class of entropy solutions. Indeed, coming back to problem (30) under assumption (A3) and looking for entropy solutions in the sense of Definition 5.2, since this time (A3) implies a control of the nonlinearity by a suitable power of $e^{t}$, say $p$, one has

$$
\int_{\Omega}|h(x, u(x))|^{\alpha} d x \leq C \int_{\Omega} e^{\alpha p u}<+\infty,
$$

where the last inequality is due to [15, Corollary 1.7]. Therefore entropy solutions are classical, and our analysis and thus Theorem 1.1 apply.

\section{References}

[1] Lorca S., Ruf B., Ubilla P. A priori bounds for superlinear problems involving the N-Laplacian. J. Differential Equations 246 (2009), no. 5, 2039-2054.

[2] Damascelli L., Pardo R. A priori estimates for some elliptic equations involving the p-Laplacian. Nonlinear Anal. Real World Appl. 41 (2018), 475-496.

[3] Brezis H., Turner R.E.L. On a class of superlinear elliptic problems. Comm. Partial Differential Equations 2 (1977), no. 6, 601-614.

[4] Gidas B., Spruck J. A priori bounds for positive solutions of nonlinear elliptic equations, Comm. Partial Differential Equations 6 (1981), no. 8, 883-901.

[5] de Figueiredo D.G., Lions P.-L., Nussbaum R.D. A priori estimates and existence of positive solutions of semilinear elliptic equations. J. Math. Pures Appl. (9) 61 (1982), no. 1, 41-63.

[6] Castro A., Pardo R. A priori bounds for positive solutions of subcritical elliptic equations. Rev. Mat. Complut. 28 (2015), no. 3, 715-731.

[7] Azizieh C., Clément P. A priori estimates and continuation methods for positive solutions of p-Laplace equations. J. Differential Equations 179 (2002), no. 1, 213-245.

[8] Ruiz D. A priori estimates and existence of positive solutions for strongly nonlinear problems. J. Differential Equations 199 (2004), no. 1, 96-114.

[9] Dall'Aglio A., De Cicco V., Giachetti D., Puel J.-P. Existence of bounded solutions for nonlinear elliptic equations in unbounded domains. NoDEA Nonlinear Differential Equations Appl. 11 (2004), no. 4, 431-450.

[10] Lorca S., Ubilla P. A priori estimate for a quasilinear problem depending on the gradient. J. Math. Anal. Appl. 367 (2010), no. 1, 69-74.

[11] Zou H.H. A priori estimates and existence for quasi-linear elliptic equations. Calc. Var. Partial Differential Equations 33 (2008), no. 4, 417-437.

[12] Damascelli L., Sciunzi B. Regularity, monotonicity and symmetry of positive solutions of $m$-Laplace equations. J. Differential Equations 206 (2004), no. 2, 483-515.

[13] Damascelli L., Sciunzi B. Harnack inequalities, maximum and comparison principles, and regularity of positive solutions of m-Laplace equations. Calc. Var. Partial Differential Equations 25 (2006), no. 2, 139-159.

[14] Brezis H., Merle F. Uniform estimates and blow-up behavior for solutions of $-\Delta u=V(x) e^{u}$ in two dimensions. Comm. Partial Differential Equations 16 (1991), no. 8-9, 1223-1253.

[15] Aguilar Crespo J.A., Peral Alonso I. Blow-up behavior for solutions of $-\Delta_{N} u=V(x) e^{u}$ in bounded domains in $\mathbb{R}^{N}$. Nonlinear Anal. 29 (1997), no. 4, 365-384. 
[16] Esposito P., Morlando F. On a quasilinear mean field equation with an exponential nonlinearity. J. Math. Pures Appl. (9) 104 (2015), no. 2, 354-382.

[17] Passalacqua T. Some applications of functional inequalities to semilinear elliptic equations Ph.D. Thesis, Università degli Studi di Milano (2015).

[18] Robert F., Wei J.-C. Asymptotic behavior of a fourth order mean field equation with Dirichlet boundary condition. Indiana Univ. Math. J. 57 (2008), no. 5, 2039-2060.

[19] Mancini G., Romani G. Uniform bounds for higher-order semilinear problems in conformal dimension. arXiv:1710.05354V3

[20] Lieberman G.M. Boundary regularity for solutions of degenerate elliptic equations. Nonlinear Anal. 12 (1988), no. 11, 12031219.

[21] Tolksdorf P. Regularity for a more general class of quasilinear elliptic equations. J. Differential Equations 51 (1984), no. 1, 126-150.

[22] Serrin J. Local behavior of solutions of quasi-linear equations. Acta Math. 111 (1964) 247-302.

[23] Allegretto W., Huang Y.X. A Picone's identity for the p-Laplacian and applications. Nonlinear Anal. 32 (1998), no. 7, 819830.

[24] Esposito P. A classification result for the quasi-linear Liouville equation. Ann. Inst. H. Poincaré Anal. Non Linéaire 35 (2018), no. 3, 781-801.

[25] Kichenassamy S., Véron L. Singular solutions of the p-Laplace equation. Math. Ann. 275 (1986), no. 4, 599-615.

[26] Royden H.L. Real Analysis. 2nd edition. Macmillan. 1968 xii+349 pp.

[27] Ren X., Wei J.-C. Counting peaks of solutions to some quasilinear elliptic equations with large exponents. J. Differential Equations 117 (1995), no. 1, 28-55.

[28] de Figueiredo D., do Ó J.M., Ruf B. Semilinear elliptic systems with exponential nonlinearities in two dimensions. Adv. Nonlinear Stud. 6 (2006), no. 2, 199-213.

[29] Bénilan P., Boccardo L., Gallouët T., Gariepy R., Pierre M., Vázquez J.L. An L $L^{1}$-theory of existence and uniqueness of solutions of nonlinear elliptic equations. Ann. Scuola Norm. Sup. Pisa Cl. Sci. (4) 22 (1995), no. 2, 241-273. 